\title{
Avaliação paicológica de PaCientes COM \\ ANOREXIA E BULIMIA NERVOSAS: INDICADORES DO \\ Método DE RoRSCHACH
}

Érika Arantes Oliveira-Cardoso ${ }^{\star}$ Manoel Antônio dos Santos ${ }^{\star}$

\section{ReSUMO}

Este estudo teve por objetivo avaliar o funcionamento lógico, afetivo e a adaptação social de pacientes com Anorexia Nervosa (AN) e Bulimia Nervosa (BN). A amostra foi composta por 27 participantes. Para coleta dos dados foi utilizado o Método de Rorschach. Os dados foram codificado e interpretados segundo as normas da Escola Francesa. Os resultados indicaram que os participantes apresentam capacidade produtiva e desejo de estabelecerem relacionamentos afetivos. Todavia, são facilmente invadidos por uma carga de afetos que não conseguem modular, o que os predispõe ao isolamento social, que gera acúmulo de inquietações e ansiedades introjetadas. A incapacidade de elaborar a tensão interna resultante os leva a escoar a ansiedade pela via somática. Evidenciou-se o comprometimento das funções psíquicas dos pacientes com AN e BN, o que indica a necessidade de acompanhamento psicoterapêutico integrado à assistência da equipe multiprofissional.

Palavras-chave: anorexia nervosa; bulimia nervosa; Método de Rorschach; avaliação psicológica; personalidade.

\section{Psychological assessment of Patients With ANOREXIA NERVOSA AND BULIMIA NERVOSA: RORSCHACH METHOD INDICATORS}

\begin{abstract}
This research aimed to assess the logical and affective functioning and social adaptation of Anorexia Nervosa (AN) and Bulimia Nervosa (BN) patients. The sample consisted of 27 participants. For data collection, the Rorschach Method

^ Psicóloga. Doutorado em Psicologia pela Universidade de São Paulo. Atualmente é psicóloga do Departamento de Psicologia e Educação da Faculdade de Filosofia, Ciências e Letras de Ribeirão Preto - Universidade de São Paulo. Endereço: Universidade de São Paulo, Faculdade de Medicina de Ribeirão Preto, Hospital das Clínicas da Faculdade de Medicina de Ribeirão Preto/USP. Av. Bandeirantes, s/n - Monte Alegre. Ribeirao Preto, SP - Brasil. CEP: 14040-901 E-mail: erikaao@bol.com.br

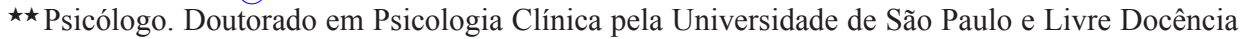
em Psicoterapia Psicanalítica pela Universidade de São Paulo, campus de Ribeirão Preto. Atualmente é Professor Associado da Faculdade de Filosofia, Ciências e Letras da Universidade de São Paulo, campus de Ribeirão Preto.

E-mail: masantos@ffclrp.usp.br
\end{abstract}


was applied and the analysis was performed according to the literature guidelines. Participants displayed productive capacity and the desire to establish affective relations. However, they are easily invaded by a load of affection they cannot modulate, which entails predisposition to social isolation, generating accumulated concerns and introjected anxieties. The inability to elaborate the resulting internal tension makes them drain their anxiety via the somatic route. This evidences that the patients' psychic functions are compromised and, consequently, that they need psychotherapy follow-up integrated with multiprofessional team care.

Keywords: anorexia nervosa; bulimia nervosa; Rorschach; psychological assessment; personality.

Os transtornos alimentares (TA) são quadros psicopatológicos que têm angariado atenção crescente por parte do meio acadêmico e do público leigo. Como elemento comum aos diversos tipos de TA observa-se grave perturbação do comportamento alimentar, que afeta, na maioria das vezes, adolescentes e adultos jovens do sexo feminino (BORGES et al., 2006). A Anorexia Nervosa (AN) e a Bulimia Nervosa (BN) constituem os tipos mais conhecidos (DOYLE; BRYANT-WAUGH, 2000).

A AN pode ser definida como uma recusa em manter o peso dentro do padrão mínimo adequado à idade e altura, acompanhada de uma perturbação no modo como o indivíduo vivencia o seu peso. Já a BN se caracteriza por episódios de comer compulsivo e por sentimento de falta de controle em relação ao comportamento alimentar. Nos dois casos o peso e o formato corporal exercem importante influência na determinação da autoestima dos pacientes (HAY, 2007).

Diversos fatores são conhecidos por contribuírem para a predisposição, instalação e manutenção dos sintomas dos TA. De um modo geral, reconhece-se a influência combinada da dinâmica familiar, do meio sociocultural e do funcionamento da personalidade do indivíduo como componentes determinantes do curso desses transtornos (OLIVEIRA; SANTOS, 2006; DUPONT; CORCOS, 2008). Estudo qualitativo evidenciou que os próprios pacientes reconhecem a importância desses fatores na etiologia da sua enfermidade (NILSSON et al., 2007). Na referida investigação, utilizou-se entrevista aberta com a seguinte questão norteadora: "Quais você acha, hoje, que foram as razões que o levaram a ter AN?". Foram obtidas como respostas as categorias: fatores familiares (como a dificuldade da família em expressar suas emoções, responsabilidades excessivas), socioculturais (culto ao corpo esbelto) e internos (elevado perfeccionismo e alto nível de exigência pessoal).

As relações familiares podem contribuir para o desencadeamento e manutenção do TA, bem como são afetadas emocionalmente pelo acometimento de um dos seus membros, gerando uma sobrecarga que aumenta a suscetibilidade dos familiares a sintomas de desgaste físico e emocional. Há farta evidência na literatura de que os TA têm impacto disruptivo sobre a organização familiar (SALBACH-ANDRAE et al., 2008). Índices de depressão maior foram significantemente 
mais elevados entre os parentes de primeiro e segundo graus dos pacientes com $\mathrm{AN}$ e BN quando comparados com parentes de indivíduos saudáveis do grupo controle (WAGNER et al., 2008).

Em geral, a literatura descreve a existência de um padrão de confusão de fronteiras nas famílias, marcado pela ausência geral de limites entre gerações e pessoas. O borramento dos limites entre eu e não-eu favorece o déficit do senso de identidade dos membros familiares. Esse déficit, por sua vez, seria determinante para o aparecimento de padrões inadequados de relacionamentos interpessoais. Por outro lado, muitas vezes essas famílias apresentam uma aparente harmonia, que escamotearia os graves conflitos subjacentes, especialmente em relação aos movimentos de individuação, diferenciação e crescimento individual. Essa padrão de relacionamento acentua o dilema vivenciado pelo membro acometido pelo TA entre prosseguir o curso de desenvolvimento ou permanecer fixado em uma posição infantil. Outros conflitos correlatos podem aparecer, como o de se submeter ao controle intergeracional versus lutar pela conquista da autonomia, ou então: priorizar metas e projetos pessoais versus corresponder às expectativas dos pais (MA, 2008).

Quanto ao ambiente sociocultural, nas últimas décadas tem aumentado a importância conferida pela sociedade ocidental à aparência física e ao padrão de beleza, o que produz a "ditadura dos corpos esbeltos" (KRELING; SANTOS, 2005; BORGES et al., 2006). Os meios de comunicação de massa tendem a perpetuar esse ideal de magreza e reforçam o estigma em relação ao ganho de peso, o que pode influenciar no desencadeamento e manutenção dos TA (LAWRIE et al., 2006; TROISE et al., 2006).

Quanto aos aspectos emocionais, a literatura mostra que o funcionamento afetivo-emocional encontra-se muito perturbado nesses quadros psicopatológicos caracterizados por grave perturbação do comportamento alimentar. Por razões peculiares, pacientes com esse tipo de transtorno relacionam à alimentação medos, ansiedades e culpas (WARD et al., 2000; BROBERG; HJALMERS; NEVONEN, 2001; CABRERA, 2006).

$\mathrm{O}$ perfil de personalidade dos pacientes com AN apresenta, tipicamente, uma constelação de características como: baixa autoestima, ansiedade elevada, perfeccionismo extremo, pensamento dicotômico e incapacidade de encontrar formas de satisfação (ABREU; CANGELLI, 2004). Segundo Santos e Peres (2006) e Connan et al. (2007), pacientes com AN apresentam acentuada fragilidade egóica, são propensos à utilização de mecanismos arcaicos de defesa, caracterizam-se por uma restrição do potencial adaptativo, controlam os próprios impulsos com excessivo rigor e tendem à passividade, introversão, obsessividade e dependência. A BN é caracterizada por episódios de ingestão alimentar exagerada, que em geral são atos secretos e rápidos, que só cessam por mal estarfísico, interrupção externa (chegada súbita de outra pessoa) ou pelos alimentos se esgotarem (AZEVEDO; ABUCHAIM, 1998). Pacientes com BN apresentam pensamentos e emoções desadaptados, autoestima flutuante e, por vezes, exibem atitudes caóticas em outros aspectos da vida, como nos estudos, na atividade 
profissional e nas relações amorosas (ABREU; CANGELLI, 2004; KEEL et al., 2000; NARDUZZI; JACKSON, 2000). A dificuldade no controle das emoções parece desempenhar um papel importante no desenvolvimento e manutenção dos sintomas (MARKEY; VANDER, 2007). Apesar de apresentarem dificuldades de ajuste interpessoal, com tendência a manterem relações afetivas instáveis, as pacientes com BN são sexualmente mais ativas do que as anoréxicas (GRABHORN et al., 2001). Em razão da propensão ao descontrole de impulsos, a atividade sexual pode assumir características de promiscuidade.

A impulsividade e agressividade seriam menos expressas nos pacientes com $\mathrm{AN}$, que tipicamente canalizam os impulsos para o próprio ego, com maior tendência à autoagressividade (MIOTTO et al., 2008). Porém, do ponto de vista da sintomatologia em nível psíquico, os TA se assemelham quanto à assinalada distorção da imagem corporal, medo mórbido de engordar, preocupação excessiva com alimentação e constante desejo não realista de emagrecimento (SILVA; SANTOS, 2006).

Diante da complexidade que caracteriza os quadros de TA, o profissional de saúde necessita obter conhecimento acerca da singularidade do funcionamento psicodinâmico, dos fenômenos psicopatológicos subjacentes a esses quadros e de suas implicações na clínica, para que possa traçar estratégias mais efetivas de intervenção (HUKE; SLADE, 2006; OLIVEIRA; SANTOS, 2006). Um dos instrumentos mais consagrados que o psicólogo tem em mãos para compreender o funcionamento psíquico de um indivíduo, considerando as condutas intelectuais, os fatores de socialização, a relação com a realidade e sua dinâmica afetiva, é o Método de Rorschach (TRAUBENBERG, 1998).

Apesar da extensa literatura disponível acerca do universo psicológico dos TA, poucos estudos têm utilizado o Rorschach na investigação da personalidade dos pacientes acometidos por esses transtornos (ROSSI et al., 2000). Para o presente estudo empreendeu-se um levantamento bibliográfico, que utilizou três bases de dados bibliográficas (MedLine, LILACS e SciELO), com o cruzamento das palavras-chave: bulimia nervosa, anorexia nervosa, transtornos alimentares e Rorschach, no período de 1997 a 2011. Foram encontrados apenas 15 artigos, dentre os quais cinco focalizavam o transtorno do comer compulsivo. Os demais serão resumidamente apresentados a seguir.

Wallach e Lowenkopf (1986) avaliaram cinco mulheres com BN, com idades entre 21 a 46 anos, por meio do Minnesota Multiphasic Personality Inventory (MMPI), Método de Rorschach e Teste de Apercepção Temática (TAT). Apesar de ser observada uma tendência a evitar relacionamentos mais profundos e dificuldade na contenção dos impulsos, os resultados indicam que os protocolos foram mais marcados pela heterogeneidade do que pela homogeneidade, sugerindo não haver uma personalidade típica para os pacientes bulímicos.

Smith et al. (1991) realizaram um estudo do tipo caso-controle com pacientes bulímicos comparados com grupo controle, objetivando avaliar possíveis diferenças nos aspectos emocionais dos dois grupos. Foram avaliados 45 pacientes com o Método de Rorschach. Os resultados mostraram que os pacientes bulimi- 
cos apresentavam maior imprecisão perceptiva, pensamento desordenado, maior vulnerabilidade à invasão dos afetos, dificuldades nos relacionamentos interpessoais e autoimagem prejudicada.

Comprometimentos semelhantes também foram encontrados por Smith, Hillard e Roll (1991), que buscaram delinear as características de personalidade que diferenciariam os adolescentes com e sem BN. Os autores aplicaram o Método de Rorschach em 12 adolescentes bulimicos e 12 do grupo controle. Os resultados sugerem que os pacientes bulimicos se mostraram mais agressivos, deprimidos e autopunitivos, tinham um pensamento mais desordenado, com imprecisões perceptivas e julgamento prejudicado. Os autores observaram que os distúrbios cognitivos não se restringiam apenas aos setores da alimentação e do peso, o que sugere uma distorção mais ampla do funcionamento intelectual.

Adolescentes com BN também foram investigados por Rossi et al. (2000). Esses autores realizaram um estudo que teve por objetivo analisar os aspectos psicopatológicos evidenciados a partir dos indicadores do Rorschach em adolescentes com AN. Foram avaliados 27 pacientes, com idades entre 11 e 14 anos (11 casos), grupo 1, e entre 15 e 18 anos (16 casos), grupo 2. Os resultados mostraram que os pacientes do grupo 1 tinham um funcionamento mais semelhante à população normal, à exceção da negação da feminilidade. Em ambos os grupos não houve indícios de depressão ou de transtorno obsessivo-compulsivo. Os autores concluíram que os pacientes mais jovens se apresentam mais preservados afetivamente e que a AN não é a expressão típica de uma determinada personalidade nem de uma estrutura psicopatológica específica.

Em relação à AN, Garcia-Alba (2004) avaliou 50 pacientes com AN do tipo restritivo, 50 pacientes deprimidos e 50 indivíduos saudáveis, com idades entre 13 e 16 anos, objetivando diagnosticar diferenças e semelhanças na personalidade dos três grupos. Utilizou como instrumento o Método de Rorschach e o MMPI. Os resultados indicaram ausência de ansiedade, bem como diferenças significativas nos sintomas depressivos e nas estratégias de enfrentamento dos pacientes com AN.

Pacientes com BN foram também comparados com pacientes com depressão e com indivíduos sem diagnóstico clínico por Weisberg, Norman e Herzog (2006). Os autores analisaram a personalidade de 57 sujeitos de cada um dos três grupos por meio do Método de Rorschach. Os resultados mostraram que, diferentemente do grupo sem diagnóstico, humor disfórico, labilidade emocional, impulsividade, vivência de sobrecarga emocional, empobrecimento da precisão perceptiva e reduzido interesse por outras pessoas foram características presentes e semelhantes nos grupos das pacientes bulimicas e com depressão. A diferença encontrada entre esses dois últimos grupos foi: maior egocentrismo e narcisismo, presença mais acentuada de raiva e negatividade nas bulimicas, ao passo que na depressão observou-se maior introspecção e mais arbitrariedade nas percepções.

Kaufer e Katz (2006) aplicaram o Método de Rorschach em 40 mulheres, sendo 20 com diagnóstico de AN e 20 sem o diagnóstico. Os resultados evidenciaram nível mais elevado de processo primário nas pacientes com diagnóstico 
de $\mathrm{AN}$, representado pelo elevado número de respostas com conteúdo sexo, sangue ou ambos, o que sugere a presença de psicopatologia em uma significativa porcentagem dessas pacientes.

Tibon e Rothschild (2009) realizaram um estudo com o objetivo de diferenciar pacientes com TA com presença de compulsão alimentar, dos pacientes com o mesmo transtorno, mas sem a compulsão. Foram avaliados, por meio do Método de Rorschach, 61 adolescentes do sexo feminino. Os resultados apontaram que a dissociação entre realidade e fantasia é mais presente nos pacientes com sintomas de compulsão alimentar.

Segundo Keel et al. (2000), mulheres que estavam livres dos sintomas bulímicos apresentaram pior ajustamento social do que as mulheres controle da comunidade, porém melhor ajustamento social do que aquelas que ainda apresentavam sintomas característicos da BN.

Recentemente, Lis et al. (2011), em estudo de caso com uma paciente com AN utilizando, dentre outras técnicas, o Método de Rorschach, destacaram como característica de personalidade da paciente: dificuldade na expressão dos afetos, prejuízos na autoimagem, limitada capacidade de introspecção e dificuldade nos relacionamentos interpessoais.

Os achados da literatura, relativos à organização e funcionamento da personalidade nos TA, sistematizados a partir da aplicação da técnica projetiva do Rorschach, são convergentes na descrição de profundo comprometimento afetivo e cognitivo que caracteriza o perfil psicológico dos pacientes, porém divergem no tocante à existência ou não de uma personalidade típica relacionada aos TA. Isso indica a necessidade de novos estudos, que permitam esclarecer as peculiaridades do funcionamento mental de pacientes com TA.

Com base nessas considerações, o presente estudo teve por objetivo avaliar o funcionamento lógico, afetivo e a adaptação social de pacientes com diagnóstico de $\mathrm{AN}$ e BN. Acredita-se que a avaliação psicodiagnóstica desses três eixos analíticos favorece a obtenção de indicadores do funcionamento da personalidade, com vistas a fornecer subsídios para que a equipe multiprofissional possa fundamentar o planejamento de intervenções mais condizentes com as necessidades psicológicas dos pacientes.

\section{Método}

\section{Participantes}

A amostra foi composta por 27 participantes, sendo 23 mulheres (85\%) e quatro homens (15\%), em atendimento junto ao Grupo de Assistência em Transtornos Alimentares (GRATA) do Hospital das Clínicas da Faculdade de Medicina de Ribeirão Preto da Universidade de São Paulo (HCFMRP-USP). A faixa etária variou dos 15 aos 23 anos de idade, sendo que a maior parte dos participantes 
tinha entre 15 e 18 anos de idade (63\%). A maioria era solteira (89\%). Em relação ao quadro clínico, mais da metade preenchia critérios diagnósticos para BN $(59 \%)$, ao passo que o restante apresentava diagnóstico de AN (41\%).

A Tabela 1 apresenta uma caracterização da amostra dos participantes em função do Índice de Massa Corporal (IMC).

Tabela 1 - Caracterização da amostra de pacientes com transtornos alimentares em função do Índice de Massa Corporal - IMC. $(\mathrm{n}=27)$

\begin{tabular}{cccc}
\hline IMC & Média & DP & Menor valor - Maior valor \\
Anorexia Nervosa & $17,7 \mathrm{Kg} / \mathrm{m}^{2}$ & 2,7 & $12,8-22,2 \mathrm{Kg} / \mathrm{m}^{2}$ \\
Bulimia Nervosa & $20,89 \mathrm{Kg} / \mathrm{m}^{2}$ & 4,2 & $15-30 \mathrm{Kg} / \mathrm{m}^{2}$ \\
\hline
\end{tabular}

Observa-se que os pacientes com AN apresentam menor Índice de Massa Corporal médio (IMC), com o menor valor individual de IMC e menor desviopadrão. Pacientes com BN, como esperado, apresentaram um IMC médio mais alto, com o maior valor individual de IMC.

\section{Cuidados éticos}

O projeto foi aprovado pelo Comitê de Ética em Pesquisa da instituição hospitalar na qual o serviço de atendimento especializado está localizado. As avaliações foram realizadas com o consentimento explícito dos participantes, após o esclarecimento dos objetivos do estudo, das questões de preservação do anonimato e sigilo em relação às informações oferecidas e do compromisso dos pesquisadores com a realização de entrevista devolutiva, que marcaria o início da assistência psicológica no serviço. As participantes deram sua anuência mediante assinatura do Termo de Consentimento Livre e Esclarecido (TCLE). No caso das participantes menores de 18 anos, o TCLE foi assinado pelos pais.

\section{Instrumento}

O Método de Rorschach ocupa posição privilegiada dentre as técnicas de avaliação de personalidade, sendo uma das mais consagradas e utilizadas em diversos países desde que foi proposta, na década de 20 do século passado. Suas possibilidades interpretativas abrem caminho para a compreensão acurada do ser humano em suas motivações profundas (PASIAN, 2000).

\section{Coleta de dados}

As avaliações foram realizadas individualmente, em uma sala reservada e com condições físicas adequadas, junto à Clinica Psicológica do Centro de Psicológica Aplicada (CPA) da Faculdade de Filosofia, Ciências e Letras de Ribeirão Preto da Universidade de São Paulo (FFCLRP-USP), em seis encontros de uma hora de duração. O primeiro encontro foi dedicado ao estabelecimento do rapport e aplicação da entrevista clínica, o segundo à aplicação do Método de Rorschach, 
no terceiro foram aplicadas as técnicas gráficas projetivas, no quarto o Teste de Apercepção Temática (TAT) e no quinto as escalas e inventários. O último encontro foi reservado para a entrevista devolutiva.

A avaliação psicodiagnóstica foi realizada com objetivo clínico e envolvia uma bateria de testes e técnicas projetivas, sendo que, no presente estudo, serão utilizados somente os dados referentes à aplicação do Método de Rorschach. $\mathrm{O}$ teste foi aplicado segundo as recomendações técnicas preconizadas pela literatura (PASIAN, 2000).

\section{Análise dos dados}

O trabalho de apuração e sistematização dos resultados da técnica projetiva aplicada foi conduzido por dois juízes, psicólogos com experiência em avaliação psicológica, de forma independente e concomitante à sua coleta. A codificação e interpretação dos dados foi realizada como preconizada pela literatura, sendo utilizada a nomenclatura da Escola Francesa (TRAUBENBERG, 1998).

Os resultados foram comparados com os dados da padronização brasileira (PASIAN, 2000) e interpretados segundo a abordagem psicodinâmica, que fundamenta o instrumento do ponto de vista teórico-metodológico. Uma vez que não há na literatura, até o presente momento, normas de padronização para adolescentes, foram utilizados os dados disponíveis para a idade adulta, que mais se aproximam da faixa etária da amostra.

\section{Resultados}

Os resultados obtidos foram organizados em três eixos: funcionamento lógico e adaptativo, funcionamento afetivo e adaptação social.

\section{Funcionamento lógico e adaptativo}

O funcionamento lógico e adaptativo indica a base da lógica e da acuidade perceptiva do indivíduo, fatores decisivos para avaliar a integridade de funções egóicas e do tipo de vínculo mantido com a realidade (TRAUBENBERG, 1998). Esses aspectos do funcionamento individual foram aferidos segundo os indicadores: produção (número de respostas), número de respostas adicionais (Rad), número de respostas movimento humano $(\mathrm{K})$ e aspectos relacionados ao controle lógico, como a porcentagem de respostas forma ( $\mathrm{F} \%$ ) e percentual de resposta forma bem vistas $(\mathrm{F}+\%)$. Esses indicadores aparecem na Tabela 2. 
Tabela 2 - Mediana de frequência e porcentagem de indicadores obtidos pelo Método de Rorschach relativos ao funcionamento lógico e adaptativo dos pacientes com $\mathrm{AN}$ e BN

\begin{tabular}{lrcccc}
\hline & \multicolumn{2}{l}{ Anorexia Nervosa } & \multicolumn{2}{c}{ Bulimia Nervosa } & \\
\hline Variáveis & Mediana & $\begin{array}{c}\text { Máximo } \\
\text { Mímino }\end{array}$ & Mediana & $\begin{array}{c}\text { Máximo - } \\
\text { Mímino }\end{array}$ & $\begin{array}{c}\text { Mediana } \\
\text { esperada }\end{array}$ \\
\hline $\mathrm{R}$ & $12 \downarrow$ & $9-15$ & $11 \downarrow$ & $7-22$ & 14 \\
$\mathrm{Rad}$ & $0 \downarrow$ & - & $0 \downarrow$ & - & 1 \\
$\mathrm{~K}$ & $2 \uparrow$ & $0-4$ & $2 \uparrow$ & $0-5$ & 1 \\
$\mathrm{~F} \%$ & $70 \uparrow$ & $30-100$ & $60 \uparrow$ & $25-100$ & $33 \%$ \\
$\mathrm{~F}+\%$ & $45 \downarrow$ & $20-71$ & $55 \downarrow$ & $20-86$ & $67 \%$ \\
\hline
\end{tabular}

Quanto à produtividade, constatou-se que os pacientes com AN e BN apresentaram um número de respostas abaixo da mediana esperada. Em ambos os grupos a tendência de protocolos com produtividade restrita é corroborada pela não utilização de respostas adicionais.

No referente ao controle lógico, observou-se um número elevado de respostas de movimento humano e de respostas com determinante formal. Em contrapartida, nos dois grupos aparece uma reduzida porcentagem de respostas formas bem vistas, com maior comprometimento do grupo constituído pelos pacientes com diagnóstico de AN.

\section{Funcionamento afetivo}

O funcionamento afetivo foi avaliado pelo tipo de vivência afetiva, segundo os indicadores da $1^{\text {a }}$ fórmula (Tipo de Ressonância Intima - TRI), $2^{\text {a }}$ fórmula (Tendências Latentes - TL) e $3^{\text {a }}$ fórmula, e pelo controle afetivo, avaliado segundo a proporção respostas de cor associadas à forma, respostas estompage associadas à forma e respostas movimento humano e pequenas cinestesias.

A TRI indica a atitude da personalidade para consigo mesma e com o meio, o modo de percepção e as ligações funcionais profundas. A TL indica a presença ou não da angústia e ansiedade (TRAUBENBERG, 1998). A terceira fórmula indica o modo como o participante reage afetivamente na situação específica do teste.

Observa-se, pela Tabela 4, que não há conflitos entre as fórmulas relativas às vivências afetivas, sendo que os resultados obtidos na TRI, TL e $3^{\text {a }}$ fórmula caracterizam as vivências afetivas dos participantes como introversiva. Tanto os indivíduos com AN como os com BN apresentaram vivência afetiva introversiva. 
Tabela 3 - Frequência de indicadores obtidos pelo Método de Rorschach relativos às vivências afetivas dos pacientes com $\mathrm{AN}$ e $\mathrm{BN}$

\begin{tabular}{lcccccc}
\hline & \multicolumn{3}{c}{ Anorexia Nervosa } & \multicolumn{3}{c}{ Bulimia Nervosa } \\
\hline \multicolumn{1}{c}{ Variáveis } & Intro & Extra & Coartado & Intro & Extra & Ambigual \\
TRI & $5 \uparrow$ & 3 & 3 & $7 \uparrow$ & 3 & 2 \\
TL & $5 \uparrow$ & 3 & 3 & $6 \uparrow$ & 2 & 4 \\
$3^{\text {a }}$ Fórmula & $7 \uparrow$ & 4 & - & $8 \uparrow$ & 2 & 2 \\
\hline
\end{tabular}

Intro: introversivo Extra: extratensivo

A Tabela 4 sistematiza os dados referentes aos indicadores de controle afetivo.

Tabela 4 - Frequência de indicadores obtidos pelo Método de Rorschach relativos ao controle afetivo de pacientes com $\mathrm{AN}$ e $\mathrm{BN}$

\begin{tabular}{lcccc}
\hline & \multicolumn{2}{c}{ Anorexia Nervosa } & \multicolumn{2}{c}{ Bulimia Nervosa } \\
\hline Variáveis & Adequada & Inadequada & Adequada & Inadequadas \\
FC:C+CF & 3 & $8 \uparrow$ & 6 & $7 \uparrow$ \\
FE: $\mathrm{E}+\mathrm{EF}$ & 4 & $7 \uparrow$ & 3 & $9 \uparrow$ \\
$\mathrm{K}: \mathrm{k}$ & $7 \uparrow$ & 4 & $7 \uparrow$ & 6 \\
\hline
\end{tabular}

Com relação à proporção de respostas cor associadas à forma $(\mathrm{FC}: \mathrm{C}+\mathrm{CF})$ e de respostas estompage associadas à forma (FE:E+EF), observa-se, pela Tabela 5 , predomínio de respostas cor associadas à imprecisão formal (inadequadas). Já a relação entre as respostas movimento humano (grandes cinestesias) e pequenas cinestesias (K:k), indica um predomínio das grandes cinestesias, de acordo com o esperado para adultos.

\section{Adaptação socialt}

A adaptação social está relacionada ao tipo de relação que o paciente consegue estabelecer com o outro. É avaliada de acordo com a frequência de respostas banais (Ban\%), porcentagem do somatório de respostas com conteúdo animal $(\Sigma \mathrm{A} \%)$ e porcentagem do somatório de respostas com conteúdo humano $(\Sigma \mathrm{H} \%)$.

Os resultados da Tabela 5 indicaram um uso elevado de respostas banais e de conteúdo animal, e um número reduzido de respostas de conteúdo humano. Tabela 5 - Mediana da porcentagem de indicadores obtidos pelo Método de Rorschach relativos à adaptação social dos pacientes com AN e BN

\begin{tabular}{cccccc}
\hline & \multicolumn{2}{c}{ Anorexia Nervosa } & \multicolumn{3}{c}{ Bulimia Nervosa } \\
\hline Variáveis & Mediana & $\begin{array}{c}\text { Maximo - } \\
\text { Mímino }\end{array}$ & Mediana & $\begin{array}{c}\text { Maximo - } \\
\text { Mímino }\end{array}$ & $\begin{array}{c}\text { Mediana } \\
\text { esperada }\end{array}$ \\
Ban \% & $33 \uparrow$ & $0-44$ & $40 \uparrow$ & $0-58$ & $29 \%$ \\
$\Sigma$ A \% & $60 \uparrow$ & $9-88$ & $42 \downarrow$ & $27-86$ & $52 \%$ \\
$\Sigma \mathrm{H} \%$ & $6 \downarrow$ & $0-84$ & $13 \downarrow$ & $0-35$ & $14 \%$ \\
\hline
\end{tabular}




\section{Discussão}

AN e BN são considerados não apenas os diagnósticos de TA mais prevalentes, como também mais complexos, uma vez que envolvem múltiplos fatores causais e prejuízos em diversas esferas da vida do indivíduo acometido. Apesar de serem reconhecidas como entidades nosológicas distintas, essas psicopatologias apresentam diversos fatores em comum, como a insatisfação corporal, o medo mórbido de ganhar peso, o pavor e/ou repulsa do alimento, além de características semelhantes de funcionamento emocional, como baixa autoestima, elevada ansiedade e dificuldades nos relacionamentos interpessoais (DUPONT; CORCOS, 2008).

No presente estudo, observou-se uma queda na produtividade ( $\mathrm{R}$ rebaixado), que pode estar relacionada a uma inibição intelectual por razões afetivas. Em relação ao controle lógico, constatou-se baixa espontaneidade e inibição acentuada, caracterizando as reações como mais impessoais, demonstrando atitudes reprimidas $(\mathrm{F} \% \uparrow)$ e uma reduzida capacidade de precisão e controle do pensamento, com dificuldade de observação e concentração.

Espera-se desses pacientes muitos erros perceptivos, que implicam em julgamento arbitrário ou displicência no exame da realidade $(\mathrm{F}+\% \downarrow)$. Esses erros podem contribuir para aumentar a dificuldade na capacidade de interação $(\operatorname{Rad} \downarrow)$, favorecendo a desadaptação, o retraimento patológico com centramento em si mesmo e introversão (K $\uparrow$ ). A presença de erros perceptivos, pensamento desordenado e julgamento prejudicado nos pacientes com TA foi também um achado obtido por Parmer (1991), Smith et al. (1991), Smith, Hillard e Roll (1991).

Walitza, Schulze e Warnke (2001) encontraram resultados parecidos, que mostraram que, apesar de apresentarem coeficiente intelectual na média, ou mesmo acima dela, pacientes com TA estão sujeitos a um montante de ansiedade e descontrole afetivo, o que prejudica a utilização eficaz dos recursos intelectuais. Essa vicissitude desempenharia um papel importante tanto no desenvolvimento como na manutenção dos seus sintomas (MARKEY; VANDER, 2007).

Em relação aos tipos de vivências afetivas, observou-se predomínio das vivências introversivas, indicando uma tendência em se preocupar com suas próprias idéias e pensamentos. Esses pensamentos são mais orientados para a vida interior, com desinteresse pelos acontecimentos externos. Esses dados indicam uma preferência pelo isolamento ou relacionamento com menores grupos e uma prevalência do mundo imaginário sobre a realidade externa.

Os indicadores relativos ao controle dos afetos indicam um bom potencial de dinamismo interno $(\mathrm{K}>\mathrm{k})$, porém marcado pela dificuldade para manter uma coordenação adequada das vivências emocionais, em função da intensidade das mesmas, em decorrência da imaturidade dos recursos internos de contenção, instabilidade emocional e dificuldade em postergar as descargas impulsivas $(\mathrm{FC}<\mathrm{C}+\mathrm{CF})$. Em relação ao controle das vivências de ansiedade e angústia, observou-se também prejuízo na capacidade de exercer o uso da razão de forma eficiente para o domínio da angústia e depressão ( $\mathrm{FE}<\mathrm{E}+\mathrm{EF})$. 
Os resultados obtidos são consistentes com a literatura, que indica que mulheres com TA, em contraposição com grupos controles, mostram características de ansiedade e padrões de evitação em seus relacionamentos com outros adultos (WARD et al., 2000).

Quanto à adaptação social, os dados obtidos sugerem dificuldade de se identificar e em entrar em contato com o outro e manter relacionamentos mais profundos $(\mathrm{H} \downarrow)$, bem como um conformismo exagerado ao pensamento coletivo, aos valores e padrões convencionais, podendo indicar necessidade de aprovação (Ban $\uparrow)$, achado semelhante ao obtido por Wallach e Lowenkopf (1986).

Dessa forma, em decorrência da mencionada instabilidade emocional e dificuldade em postergar as descargas impulsivas, os relacionamentos interpessoais são temidos, porém, ao mesmo tempo, são marcados por um desejo intenso de aprovação social (CONNAN et al., 2007). Não foram constatadas diferenças nesse aspecto entre pacientes com $\mathrm{AN}$ e $\mathrm{BN}$.

Essa ambivalência nas relações interpessoais, marcada pela dificuldade e necessidade de entrar em contato com o outro, também foi reportada por Narduzzi e Jackson (2000), que apontaram para a coexistência de dois tipos de comportamentos em pacientes com TA: de um lado, a necessidade de manter um sentimento de separação e independência em relação ao outro e, de outro lado, a necessidade de preservarem esses relacionamentos como fonte de sustentação de sua autoestima rebaixada.

Comportamento semelhante a esse padrão de evitação-aproximação, apresentado em relação aos relacionamentos interpessoais, marcados pela ambivalência entre desejo e medo, estaria relacionado às inquietações internas e alto nível de ansiedade (OLIVEIRA; SANTOS, 2006). Esse padrão poderia estar representado também na relação estabelecida com o alimento, caracterizada pela alternância entre os movimentos de fusão e rejeição da alimentação (DUPONT; CORCOS, 2008).

O desenvolvimento da insatisfação com o próprio corpo poderia estar relacionado com a alta expectativa de serem rejeitadas pelo outro. Esse temor faria com que tais indivíduos dependessem exageradamente da aprovação social e da aceitação dos outros, tornando-os mais sensíveis às mensagens da sociedade relacionadas à aparência e aos valores positivos associados à magreza (TROISE et al., 2006).

No contato social os pacientes com AN e BN apresentaram comportamentos diferentes. Na AN observa-se uma maior imaturidade no processo de contato com a realidade, uma percepção mais intuitiva e menos elaborada e uma estereotipia do pensamento $(\mathrm{A} \uparrow)$. Na $\mathrm{BN}$ constatou-se incoerência e dispersão do pensamento, dificuldade em lidar com o outro e de seguir as regras e normas sociais (A $\downarrow$ ).

De acordo com a literatura, a impulsividade e agressividade seriam menos expressas nos pacientes com $\mathrm{AN}$ do que nos pacientes com $\mathrm{BN}$; nos primeiros haveria maior tendência à autoagressividade, dada à incapacidade de expressão aberta de sua hostilidade (MIOTTO et al., 2008). 
Os resultados de $\mathrm{K}$ elevado, somados ao rebaixamento de $\mathrm{H}$ e ao predomínio da introversão, indicam uma dificuldade dos pacientes em voltarem a atenção para o mundo exterior, sendo seus interesses e comportamentos direcionados preferencialmente para o mundo interno, o que dificulta o estabelecimento e manutenção de contatos sociais, ao mesmo tempo em que favorece a relação obsessiva com o peso e a forma corporal.

\section{CONSIDERaÇões Finais}

De acordo com os achados da presente investigação, os indivíduos com diagnóstico de $\mathrm{AN}$ e $\mathrm{BN}$ avaliados apresentaram capacidade produtiva inibida por fatores afetivos. Constatou-se que eles preservavam seu desejo de estabelecerem relacionamentos afetivos, todavia, eram facilmente invadidos por uma carga maciça de afetos que não conseguem controlar e modular, o que os predispõe ao isolamento social, que por sua vez gera acúmulo de inquietações e ansiedades introjetadas. A incapacidade de elaborar a tensão interna resultante os leva a escoar a ansiedade pela via somática, por meio de comportamentos autoagressivos e somatizações.

Um dado positivo, de acordo com a literatura, é o de que a melhora dos sintomas dos TA vem acompanhada por uma melhor qualidade dos relacionamentos sociais. Segundo Keel et al. (2000), mulheres que estavam livres dos sintomas bulímicos apresentaram pior ajustamento social do que o grupo controle, porém melhor ajustamento social do que aquelas que ainda apresentavam sintomas característicos da $\mathrm{BN}$.

Diante desses resultados, evidencia-se o comprometimento das funções psíquicas dos pacientes com TA e a consequente necessidade de um acompanhamento psicoterapêutico. O suporte psicológico, segundo Huke e Slade (2006), deveria se estender aos indivíduos do círculo relacional mais próximo ao paciente, em especial os familiares, a fim de que seja encorajado um diálogo mais aberto a respeito das necessidades subjetivas dos membros acometidos pelo TA, de modo a atenuar suas dificuldades relacionais.

\section{REFERÊNCIAS}

ABREU, C. N.; CANGELLI, R. Anorexia nervosa e bulimia nervosa: abordagem cognitiva -construtivista de psicoterapia. Revista de Psiquiatria Clínica, [S.1.], v. 31, n. 4, p. 177-183, 2004.

AZEVEDO, A. M. C.; ABUCHAIM, A. L. G. Bulimia nervosa: classificação diagnóstica e quadro clínico. In: NUNES, M. A. A. et al. (Org.). Transtornos alimentares e obesidade. Porto Alegre: Artmed, 1998. p. 31-49.

BORGES, N. J. B. G. et al. Transtornos alimentares: quadro clínico. Medicina, Ribeirão Preto, v. 39, n. 3, p. 340-348, 2006. 
BROBERG, A. G.; HJALMERS, I.; NEVONEN, L. Eating disorders, attachment and interpersonal difficulties: a comparison between 18-24 year old patients and controls. European Eating Disorders Review, [S.1.], v. 9, n. 1, p. 381-396, 2001.

CABRERA, C. C. Estratégias de intervenção interdisciplinar no cuidado com o paciente com transtorno alimentar: o tratamento farmacológico. Medicina, Ribeirão Preto, v. 39, n. 3, p. 375-80, 2006.

CONNAN, F. et al. Poor social comparison and the tendency to submissive behavior in anorexia nervosa. International Journal of Eating Disorders, [S.1.], v. 40, n. 8, p. 733-739, 2007.

DOYLE, J.; BRYANT-WAUGH, R. Epidemiology. In: LASK, B.; BRYANTWAUGH, R. (Ed.). Anorexia nervosa and related eating disorders in childhood and adolescence. East Sussex: Psychology, 2000. p. 41-61.

DUPONT, M. E.; CORCOS, M. Psychopathology in eating disorders: new trends. La Revue du Praticien, [S.1.], v. 58, n. 2, p. 141-149, 2008.

GARCIA-ALBA, C. Anorexia and depression: depressive comorbidity in anorexic adolescents. The Spanish Journal of Psychology, [S.1.], v. 7, n. 1, p. 40-52, 2004.

GRABHORN, R. et al. Social anxiety in anorexia and bulimia nervosa: the mediating role of shame. Clinical Psychology and Psychotherapy, [S.1.], v. 13, n. 1, p. 12-19, 2001.

HAY, P. J. Understanding bulimia. Australian Family Physician, [S.1.], v. 36, n. 9, p. 708-731, 2007.

HUKE, K.; SLADE, P. An exploratory investigation of the experiences of partners living with people who have bulimia nervosa. European Eating Disorders Review, [S.1.], v. 14, n. 1, p. 436-447, 2006.

KAUFER, J. F; KATZ, J. L. Rorschach responses in anorectic and nonanorectic women. International Journal of Eating Disorders, [S.1.], v. 3, n. 1, p. 65-74, 2006.

KEEL, P. K. et al. Social adjustment over 10 years following diagnosis with bulimia nervosa. International Journal of Eating Disorders, [S.1.], v. 27, n. 1, p. 21-28, 2000.

KRELING, D. B.; SANTOS, M. A. Anorexia nervosa: um relato de atendimento clínico realizado em contexto multidisciplinar. In: SANTOS, M. A.; SIMON, C. P.; MELO-SILVA, L. L. (Org.). Formação em Psicologia: processos clínicos. São Paulo: Vetor, 2005. p. 143-162. 
LAWRIE, Z. E. et al. Media Influence on the Body Image of Children and Adolescents. Eating Disorders, [S.1.]v. 14, n. 1, p. 355-364, 2006.

LIS, A. et al. Attachment, assessment, and psychological intervention: a case study of anorexia. Journal of Personality Assessment, [S.1.], v. 93, n. 5, p. 434444, 2011.

MA, J. L. Eating disorders, parent-child conflicts, and family therapy in Shenzhen, China. Quality Health Research, [S.1.], v. 18, n. 6, p. 803-810, 2008.

MARKEY, M. A.; VANDER, W. J. S. The role of emotional intelligence and negative affect in bulimic symptomatology. Comprehensive Psychiatry, [S.l.], v. 48, n. 5, p. 458-464, 2007.

MIOTTO, P. et al. Aggressiveness, anger, and hostility in eating disorders. Comprehensive Psychiatry, [S.1.], v. 49, n. 4, p. 364-373, 2008.

NARDUZZI, K. J.; JACKSON, T. Personality differences between eatingdisordered women and a nonclinical comparison sample: a discriminant classification analysis. Journal of Clinical Psychology, [S.1.], v. 56, n. 6, p. 699$710,2000$.

NILSSON, K. et al. Causes of adolescent onset anorexia nervosa: Patient perspectives. Eating Disorders, v. 15, n. 1, p.125-133, 2007.

OLIVEIRA, E. A.; SANTOS, M. A. Perfil psicológico de pacientes com anorexia e bulimia nervosas: a ótica do psicodiganóstico. Medicina, Ribeirão Preto, v. 39, n. 3, p. 353-360, 2006.

PARMER, J. C. Bulimia and object relations: MMPI and Rorschach variables. Journal of Personality Assessment, [S.1.], v. 56, n. 2, p. 266-276, 1991.

PASIAN, S. R. O psicodiagnóstico de Rorschach em adultos: atlas, normas e reflexões. São Paulo: Casa do Psicólogo, 2000.

ROSSI, G. et al. Rorschach and anorexia nervosa: a study of 27 cases. Minerva Psichiatrica, [S.1.], v. 41, n. 4, p. 205-222, 2000.

SALBACH-ANDRAE, $\mathrm{H}$. et al. Correspondence between self-reported and parent-reported psychopathology in adolescents with eating disorders. Psychopathology, [S.1.], v. 41, n. 5, p. 307-312, 2008.

SANTOS, M. A.; PERES, R. S. Contribuições do desenho da figura humana para avaliação da imagem corporal na anorexia nervosa. Medicina, Ribeirão Preto, v. 3, n. 39, p. 361-370, 2006. 
SILVA, L. M; SANTOS, M. A. Construindo pontes: relato de experiência de uma equipe multidisciplinar em transtornos alimentares. Medicina, Ribeirão Preto, v. 3, n. 3, p. 415-424, 2006.

SMITH J. E.; HILLARD, M. C.; ROLL, S. Rorschach evaluation of adolescent bulimics. Adolescence, [S.1.], v. 26, n. 103, p. 687-696, 1991.

SMITH, J. E. et al. Rorschach assessment of purging and nonpurging bulimics. Journal of Personality Assessment, [S.1.], v. 56, n. 2, p. 277-288, 1991.

TIBON, S.; ROTHSCHILD, L. Dissociative states in eating disorders: an empirical Rorschach study. Psychoanalytic Psychology, [S.1.], v. 26, n. 1, p. 6982, 2009.

TRAUBENBERG, N. R. A prática do Rorschach. São Paulo: Vetor, 1998.

TROISE, A. et al. Body dissatisfaction in women with eating disorders: relationship to early separation anxiety and insecure attachment. Psychosomatic Medicine, [S.1.], v. 68, n. 1, p. 449-453, 2006.

WAGNER, A. et al. Mental disorders among relatives of patients with anorexia nervosa and bulimia nervosa. Zeitschrift für Kinder - und Jugendpsychiatrie und Psychotherapie, [S.1.], v. 36, n. 3, p. 177-184, 2008.

WALITZA, S.; SCHULZE, U.; WARNKE, A. Differences between adolescent patients with anorexia and bulimia nervosa with reference to psychological and psychosocial markers. Zeitschrift für Kinder - und Jugendpsychiatrie und Psychotherapie, [S.1.], v. 29, n. 2, p. 117-125, 2001.

WALLACH, J. D.; LOWENKOPF E. L. Five bulimic women: MMPI, Rorschach, and TAT characteristics. International Journal of Eating Disorders, [S.1.], v. 3, n. 4, p. 53-66, 1986.

WARD, A. et al. Attachment patterns in eating disorders: past in the present. International Journal of Eating Disorders, [S.1.], v. 28, n. 4, p. 370-376, 2000.

WEISBERG, L. J.; NORMAN, D. K.; HERZOG, D. B. Personality functioning in normal weight bulimia. International Journal of Eating Disorders. [S.1.], v. 6, n. 5, p. 615-631, 2006.

Recebido em: 22 de abril de 2010 Aceito em: 22 de fevereiro de 2012 